\title{
Unification in Logic
}

\author{
Rosalie Iemhoff \\ Department of Philosophy, \\ Utrecht University \\ Rosalie.Iemhoff@phil.uu.nl
}

There are many problems in mathematics that can be cast in terms of unification, meaning that a solution of the problem is a substitution that identifies two terms, either literally, or against a background theory of equivalence. In the context of logics, a unifier is a substitution under which a formula becomes derivable in the logic.

In classical propositional logic, all unifiable formulas have a most general unifier, which is a unifier that generates all other unifiers of a formula. Nonclassical logics in general do not have this useful property, but many modal and intermediate propositional logics satisfy a slightly weaker property. In these logics, for every formula there is a finite set of unifiers such that any other unifier of the formula is generated by one of them.

The study of unification in nonclassical logics mainly uses semantical techniques. Even though there exist algorithms to find unifiers, proofs of correctness again use semantics. In this talk a purely syntactic treatment of unification is presented, and it is shown that most known results can be obtained, and in some cases extended, by this approach. 